\title{
Orbital-selective Mott transitions in two-band Hubbard models
}

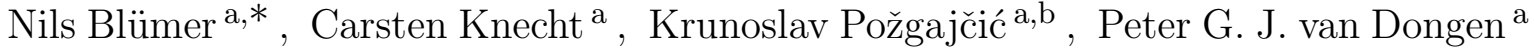 \\ ${ }^{\mathrm{a}}$ Institute of Physics, Johannes Gutenberg University, 55128 Mainz, Germany \\ ${ }^{\mathrm{b}}$ Institute for Theoretical Physics, J. W. Goethe University, 60438 Frankfurt/Main, Germany \\ Received 7 June 2006
}

\begin{abstract}
' The anisotropic two-orbital Hubbard model is investigated at low temperatures using high-precision quantum Monte Carlo (QMC) simulations within dynamical mean-field theory (DMFT). We demonstrate that two distinct orbital-selective Mott transitions ' (OSMTs) occur for a bandwidth ratio of 2 even without spin-flip contributions to the Hund exchange, and we quantify numerical ' errors in earlier QMC data which had obscured the second transition. The limit of small inter-orbital coupling is introduced via a ' new generalized Hamiltonian and studied using QMC and Potthoff's self-energy functional method, yielding insight into the nature of the OSMTs and the non-Fermi-liquid OSM phase and opening the possibility for a new quantum-critical point.
\end{abstract}

PACS: 71.10.Fd; 71.27.+; 71.30.+h

Keywords: Mott transition, orbital-selective, Hubbard model

\section{Introduction}

Recently, the suggestion [1] that the two consecutive phase transitions experimentally observed [2] in $\mathrm{Ca}_{2-x} \mathrm{Sr}_{x} \mathrm{RuO}_{4}$ should be interpreted as "orbital-selective Mott transitions" (OSMTs) sparked a cascade of related theoretical papers (see [3] and references therein).

Microscopic studies of OSMTs usually consider the 2band Hubbard model $H=H_{1}+H_{2}+H_{3}$, where

$H_{1}=-\sum_{\langle i j\rangle m \sigma} t_{m} c_{i m \sigma}^{\dagger} c_{j m \sigma}+U \sum_{i m} n_{i m \uparrow} n_{i m \downarrow}$

$H_{2}=\sum_{i \sigma \sigma^{\prime}}\left(U^{\prime}-\delta_{\sigma \sigma^{\prime}} J_{z}\right) n_{i 1 \sigma} n_{i 2 \sigma^{\prime}}$

include hopping between nearest-neighbor sites $i, j$ with amplitude $t_{m}$ for orbital $m \in\{1,2\}$, intra- and interorbital Coulomb repulsion parametrized by $U$ and $U^{\prime}$, respectively, and Ising-type Hund's exchange coupling; $n_{i m \sigma}=c_{i m \sigma}^{\dagger} c_{i m \sigma}$ for spin $\sigma \in\{\uparrow, \downarrow\}$. In addition,

$$
H_{3}=\frac{1}{2} J_{\perp} \sum_{i m \sigma} c_{i m \sigma}^{\dagger}\left(c_{i \bar{m} \bar{\sigma}}^{\dagger} c_{i m \bar{\sigma}}+c_{i m \bar{\sigma}}^{\dagger} c_{i \bar{m} \bar{\sigma}}\right) c_{i \bar{m} \sigma}
$$

contains spin-flip and pair-hopping terms (with $\overline{1} \equiv 2, \bar{\uparrow} \equiv \downarrow$ etc.). As in [3], we refer to $H_{1}+H_{2}+H_{3}$ with the isotropic coupling $J_{z}=J_{\perp} \equiv J$ as the $J$-model and to the simplified

\footnotetext{
* Corresponding author.

Email address: Nils.Bluemer@uni-mainz.de (Nils Blümer).

URL: komet337.physik.uni-mainz.de/Bluemer/ (Nils Blümer).
}
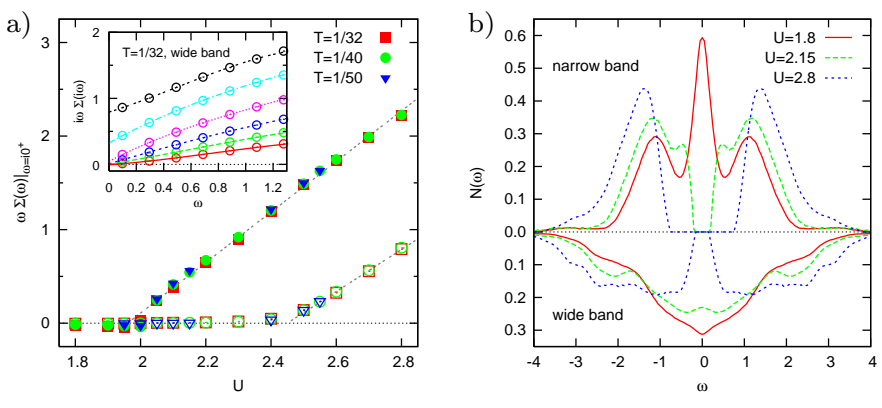

Fig. 1. a) Low-frequency analysis of self-energy. Main panel: weight of singularity at $\omega=0$ for narrow/wide band (filled/open symbols) as determined by polynomial fits [shown in inset for $U=2.8,2.6,2.4,2.2,2.0,1.8$ (top to bottom)]. b) Spectra for metallic, orbital-selective, and insulating phases.

Hamiltonian $H_{1}+H_{2}$ as the $J_{z}$-model; unless noted, $U^{\prime}=$ $U / 2, J=U / 4$ so that $U^{\prime}+2 J=U$.

Early theoretical studies suggested that the expected 2 distinct OSMTs occur only in the full $J$ model [4], but not in the Ising type $J_{z}$-model $[5,6]$. Thus, it seemed as if spinflip and pair-hopping terms were essential ingredients to orbital-selective physics. However, this is not actually the case, as shown in a low-frequency analysis of high-precision QMC data [3] in Fig. 1a: a singularity develops at $\omega=0$ for $U_{c 1} \approx 2.0$ in the narrow-band self-energy, but only at $U_{c 1} \approx 2.5$ in the wide-band self-energy (with linear increase for $U>U_{c 1 / 2}$ ). Corresponding spectra (Fig. 1b) illustrate the characteristics of the 3 distinct phases. 

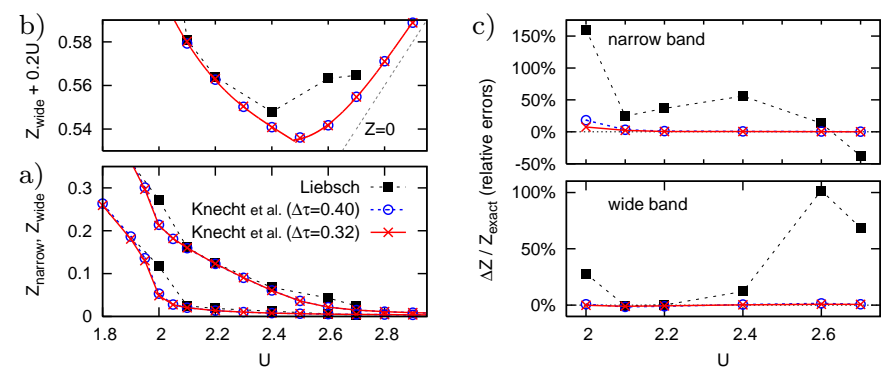

Fig. 2. a) QMC estimates of quasiparticle weights $Z$ at $T=1 / 32$ : a) high-precision data [3] (cirles, crosses) clearly shows kinks at $U_{c 1} \approx 2.0 ;$ b) suitable analysis reveals a second kink at $U_{c 2} \approx 2.5$. The second transition is lost in the noise of earlier data [5] (squares) with errors exceeding $100 \%$ at both transitions (c).

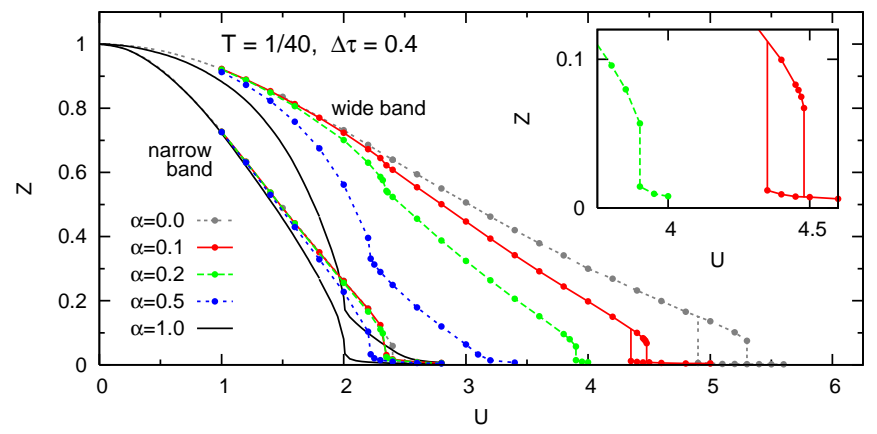

Fig. 3. Quasiparticle weights for generalized two-orbital model: at $T=1 / 40$, the wide-band transition remains first order (with hysteresis) for small enough inter-orbital coupling $\alpha \lesssim 0.2$.

Discrete estimates $Z=[1-\operatorname{Im} \Sigma(i \pi T) /(\pi T)]^{-1}$ for the quasiparticle weight clearly show (only) the narrow-band transition (Fig. 2a); a second (wide-band) transition is visible as kink only in high-precision QMC data (circles, crosses) and after adding a linear term (Fig. 2b). Possible signals from this transitions cannot be distinguished from numerical errors in earlier QMC data [5] (squares) since they exceed $100 \%$ at both transitions (Fig. 2c).

\section{Limit of small inter-orbital coupling}

Clearly, the resolution of two orbital-selective Mott transitions with critical interactions differing by only about $20 \%$ is a very challenging task, in particular at temperatures attainable using QMC. In this situation, much insight can be gained by abandoning the constraint $U^{\prime}+2 J=U$ fulfilled in nearly all earlier studies and instead studying the limit of small inter-orbital coupling. Hence, we consider $H=$ $H_{1}+\alpha H_{2}$ with $0 \leq \alpha \leq 1$ so that $\alpha=0$ corresponds to uncoupled orbitals and $\alpha=1$ to the case studied previously.

It is a priori clear, that for $\alpha=0$ each orbital should undergo a usual Mott transition at an interaction determined by the corresponding bandwidth $(W=2$ for the narrow, $W=4$ for the wide band); note however, that even in this case the QMC results for both orbitals have no scaling relation at fixed $T>0$. This is seen in Fig. 3: For $\alpha=0$, a large hysteresis region appears in the wide-band quasiparticle weight (uppermost, grey line) while only a single coex-
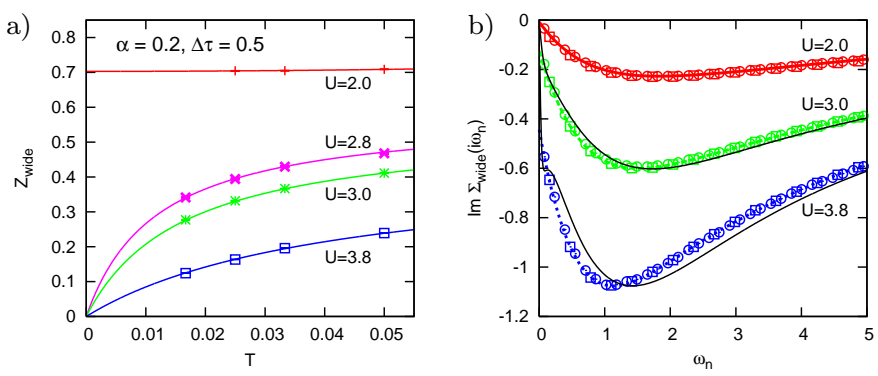

Fig. 4. a) Discrete QMC estimates of wide-band quasiparticle weights for $\alpha=0.2$. In OSM phase $(2.3 \lesssim U \lesssim 3.9), Z_{\text {wide }}$ decays to 0 for $T \rightarrow 0$. b) In contrast, the self-energy is practically $T$-independent in QMC (squares: $T=1 / 20$, circles: $T=1 / 40$ ), consistent with DIA for $T=0$ (solid lines).

istence point is resolved for the narrow band (at $T=1 / 40$ ). Very importantly, the wide-band transition evidently remains first order at small, but significant inter-orbital coupling ( $\alpha=0.1, \alpha=0.2)$. It may be expected that the firstorder range (in $\alpha$ ) increases at lower temperatures which suggests that the wide-band OSMT might be very weakly first order even at $\alpha=1$. However, the alternative of a quantum phase transition at some critical value $\alpha=\alpha_{c}$ is equally interesting and warrants further investigation.

\section{Nature of orbital-selective Mott phase}

As shown above, the (discretely estimated) quasiparticle weight $Z$ is not well suited for detecting the second OSMT. In fact, it is even misleading in the OSM phase: as seen in Fig. 4a, $Z_{\text {wide }}$ is nearly constant as a function of $T$ in the metallic Fermi-liquid phase $(U=2.0)$. However, this observable decays to 0 for $T \rightarrow 0$ in the OSM phase. Naively, one might conclude that the OSM phase becomes indistinguishable from the insulating phase for $T=0$. However, Fig. $4 \mathrm{~b}$ proves that this is not the case: the self-energies are practically $T$-independent both in the metallic and in the OSM phase [with agreement between QMC results for different $T$ and self-energy functional theory in dynamical impurity approximation (DIA)]; thus, the $T$ dependence in $Z_{\text {wide }}$ is an artifact of the discrete approximation. The nonFermi-liquid character of the wide band in the OSM phase is clearly seen as the finite limit of $\operatorname{Im} \Sigma$ for $\omega \rightarrow i 0^{+}$.

We acknowledge support by the DFG (FG 559, Bl775/1).

\section{References}

[1] V.I. Anisimov, I.A. Nekrasov, D.E. Kondakov, T.M. Rice, and M. Sigrist, Eur. Phys. J. B 25, 191 (2002).

[2] S. Nakatsuji, Y. Maeno, Phys. Rev. Lett. 84, 2666 (2000); Phys. Rev. B 62, 6458 (2000).

[3] C. Knecht, N. Blümer, and P.G.J. van Dongen, Phys. Rev. B 72, 081103(R) (2005); P. G. J. van Dongen, C. Knecht, and N. Blümer, phys. stat. sol. (b) 243, 116 (2006).

[4] A. Koga, N. Kawakami, T.M. Rice, and M. Sigrist, Phys. Rev. Lett. 92, 216402 (2004).

[5] A. Liebsch, Phys. Rev. B 70, 165103 (2004).

[6] A. Koga, N. Kawakami, T. Rice, and M. Sigrist, Physica B 359361, 1366 (2005). 